\title{
Pathological investigation of deaths following surgery, anaesthesia, and medical procedures
}

\author{
Roger D Start, Simon S Cross
}

\begin{abstract}
The pathological investigation of deaths following surgery, anaesthesia, and medical procedures is discussed. The definition of "postoperative death" is examined and the classification of deaths following procedures detailed. The review of individual cases is described and the overall approach to necropsy and interpretation considered. There are specific sections dealing with the cardiovascular system (including air embolism, perioperative myocardial infarction, cardiac pacemakers, central venous catheters, cardiac surgery, heart valve replacement, angioplasty, and vascular surgery); respiratory system (postoperative pneumonia, pulmonary embolism, pneumothorax); central nervous system (dissection of cervical spinal cord), hepatobiliary and gastrointestinal system; musculoskeletal system; and head and neck region. Deaths associated with anaesthesia are classified and the specific problems of epidural anaesthesia and malignant hyperthermia discussed. The article concludes with a section on the recording of necropsy findings and their communication to clinicians and medicolegal authorities.

(F Clin Pathol 1999;52:640-652)
\end{abstract}

Keywords: postoperative deaths; anaesthetic related deaths

Histopathology,

Chesterfield and North

Derbyshire Royal

Hospital NHS Trust,

Calow, Chesterfield

S44 5BL, UK

R D Start

Department of Pathology, Sheffield

University Medical

School, Beech Hill

Road, Sheffield

S10 2RX, UK

S S Cross

Correspondence to: Dr Start.

Accepted for publication 17 June 1999 after a surgical operation, invasive diagnostic procedure, or an anaesthetic. Although ther-
Specific problems at necropsy on deaths associated with surgery and anaesthesia

Morphological findings, particularly in anaesthetic-related deaths, may be minimal or absent

The necropsy may be technically difficult because of the surgical intervention and its sequelae

Numerous surgical and anaesthetic devices may have been introduced into the patient during the procedure

Interpretation is difficult in multifactorial deaths Anxiety in clinicians about medicolegal implications

Common fatal complications of invasive procedures

Sepsis, including bronchopneumonia

Multi-organ failure including adult respiratory distress syndrome (often secondary to sepsis)

Pulmonary embolism

Perioperative myocardial infarction

Haemorrhage

eview is relevant to pathologists in all countries, the specific medicolegal details are mainly related to the coronial system of England and Wales.

\section{Terminology}

The term "postoperative" is vague and deaths resulting from invasive diagnostic procedures and anaesthesia are often not reported to the appropriate authority because clinicians of all grades are unaware of their legal responsibilities. A better system is to classify deaths into those associated with invasive procedures, including invasive diagnostic procedures, and those associated with anaesthesia. ${ }^{1}$ Such deaths may be further classified into four subgroups according to the cause of death:

(1) Those directly caused by the disease or injury for which the invasive procedure or anaesthesia was being performed.

(2) Those caused by a disease or abnormality other than that for which the procedure was being performed.

(3) Those resulting from a mishap during, or a complication of, the invasive procedure. 
(4) Those resulting from a mishap during, or as a complication of, the administration of an anaesthetic.

\section{Classification of deaths associated with} invasive procedures and anaesthesia

A Those directly caused by the disease or injury for which the invasive procedure or anaesthesia was being performed

B Those caused by a disease or abnormality other than that for which the procedure was being performed

C Those resulting from a mishap during, or a complication of, the invasive procedure

D Those resulting from a mishap during, or as a complication of, the administration of an anaesthetic

Precse classification of individual cases may only be possible after full investigation.

Necropsies on patients who have died in the postoperative period have a central role in medical audit ${ }^{2-16}$ and data from necropsy reports are of prime importance to national organisations seeking to improve perioperative care, such as the National Confidential Enquiry into Perioperative Deaths. ${ }^{17-20}$ These necropsies present different problems from those on subjects who have died outside hospital or inside hospital without operative intervention in both dissection technique and interpretation of findings. There are many pitfalls of which pathologists should be aware, although most can be avoided by a thorough review of the case before starting the necropsy.

\section{Review of the case}

The hospital notes should always be obtained before starting a necropsy, and radiographs may add useful information, particularly if any diagnostic imaging has been performed during the operation-for example, intraoperative cholangiograms in biliary surgery, or as part of a procedure such as coronary artery angioplasty. Video recording of operative procedures is becoming widespread and viewing of such recordings might be appropriate in some cases. The notes should be read carefully, concentrating on the current admission and viewing all relevant documentation including the operation record, the anaesthetic record, and laboratory reports. The nursing notes can also provide helpful information where the medical notes are sparse. If the case is complicated with a lengthy hospital stay then it is often useful to construct a succinct summary with the dates and timing of all significant events. All pathologists may be faced with necropsies following surgical procedures not usually performed in their own institutions. If there are any matters that require clarification it may be prudent to contact the relevant clinician before starting the necropsy, or alternatively to seek the technical advice and expert opinion of an independent clinical consultant who is unconnected with the hospital, and who is not a colleague of the team involved in the incident.
The medicolegal status of the necropsy When the case notes have been reviewed, the medicolegal status of the necropsy should be considered. ${ }^{21}$ The case will have been presented to the pathologist either as a consent case, with "consent" (in England and Wales this would be a record of absence of objection under the 1961 Human Tissue Act ${ }^{22}{ }^{23}$ from the nearest relatives, or as a formal medicolegal case under the jurisdiction of the coroner or equivalent authority. If the necropsy has been presented as a consent case then the details of the case should be reviewed to ensure that the case should not have been referred to the appropriate medicolegal authority. ${ }^{24} 25$ Under the coronial system in England and Wales there is a common law duty to report any cases which should be considered by the coroner, so a pathologist is obliged to report any such case. Direct referral to a medicolegal authority without contacting the clinicians who obtained "consent" for the necropsy is discourteous and will lead to ill feeling and a lack of trust, so it is always wise to contact the clinicians and ask them to refer the case to the appropriate medicolegal authority. The reasons for referring a case to a medicolegal authority will vary according to the local legislation of that authority but the broad categories (as described in the coronial system of England and Wales) which could apply to postoperative deaths include:

- Deaths occurring during an operation or before recovery from an operation (often taken to be recovery from the anaesthetic but can relate to any aspect of recovery).

- Allegations of negligence and complaints concerning quality of care.

- Suspicious circumstances.

- Deaths related to industrial disease or industrial accidents.

- Deaths from accidents, for example road traffic accidents.

- Deaths related to abortion.

It can be seen that many postoperative deaths will fall into one of these categories so that most postoperative deaths in England and Wales will be performed under instructions from coroners. In Scotland the investigation of such deaths will be directed by the Procurator Fiscal and in other countries by appropriate medicolegal authorities, such as the medical examiner.

Who should perform the necropsy?

This question also relates to the medicolegal context in which the necropsy is being performed. In England and Wales there is a requirement under the Coroner's Rules that independent pathologists should perform necropsies in postoperative deaths where there are allegations of negligence, and similar requirements exist in most other medicolegal systems. This is obviously a wise precaution so that the impartiality of the pathologist cannot be called into question. Such arrangements also prevent any professional embarrassment involved in criticising close colleagues. In some centres this arrangement has been extended so that all postoperative necropsies are performed 
by independent pathologists, usually forensic pathologists. While allegations of negligence can arise after the necropsy has been performed, there will be many necropsies on postoperative deaths which never have allegations of negligence and can be performed by the pathologists in the hospital where the patient was treated, on the direction or with the consent of the relevant legal authority. This has the advantages of timeliness of the necropsy in centres distant from other pathologists and potentially closer clinicopathological correlation of the findings. Individual pathologists must consider the appropriateness of their involvement on a case by case basis. Circumstances can occur where during the course of the necropsy it becomes apparent that there may be subsequent allegations of negligence, for example the discovery of a clip across the common bile duct or a retained swab or instrument. In such situations it may be appropriate for the pathologist to halt the necropsy, preserve the evidence, and contact the local medicolegal authority, after which another pathologist (usually a forensic pathologist) may be called in to view the findings and complete the examination.

\section{Preparation for necropsy}

Proper review of the case should provide the pathologist with sufficient understanding to identify the central issues for investigation, and uncertainty suggests that further review and consultation may be required. Necropsies on deaths associated with surgery and anaesthesia raise specific problems. There may be few if any gross morphological findings, and the necropsy may be technically difficult as a result of the surgical intervention and its sequelae. Exudates, sepsis, adhesions, haemorrhage, and oedema may all distort normal anatomy, and it is essential to know the full details of any previous procedures. Consideration should be given to the retention of antemortem body fluid and blood samples. ${ }^{26}$ It is worth remembering that while clinical chemistry and haematology laboratories may dispose of samples quickly, blood transfusion and serology laboratories retain samples for days or weeks as a routine. These may be useful for the confirmation of disseminated intravascular coagulation, blood transfusion errors, or the assessment of creatine phosphokinase activity in malignant hyperthermia. Similarly, recent and previous histology specimens should be reviewed with the reporting pathologist. Requests for results of laboratory test taken during life but not available at the time of death can be worthwhile. Cardiac enzyme levels and blood cultures may provide useful clues to the cause of death but interpretation may require expert assistance. ${ }^{27}$

Numerous medical devices may have been introduced into the patient, such as nasogastric tubes, urinary catheters, wound drains, chest drains, subcutaneous pacemakers, and metal or plastic prostheses. It is essential that none of these is removed before necropsy, and pathologists must ensure that prospective arrangements with wards, intensive care units, imaging departments, and operating theatres prevent removal. Placement and patency of all such devices may need to be confirmed at necropsy. A similar approach is necessary for all anaesthetic devices. Mishaps may occur when multiple tubes are present, when a prescribed agent may be given into the wrong tube, for example gastric medicines into a central venous line.

\section{The necropsy dissection}

\section{EXTERNAL EXAMINATION}

The site and type of all anaesthetic and surgical devices should be recorded, preferably on a body chart. Similarly, the sites, lengths, and ages of all surgical wounds and scars, including colostomy or ileostomy sites, should be recorded using appropriate anatomical descriptions and absolute measurements.

The degree of oedema, jaundice, and cyanosis should be noted, together with the state and nature of any dentition present. Skin rashes are common and may be significant, particularly in relation to drug reactions, septicaemia, internal malignancies, and immunosuppression. All areas of bruising should be recorded and locations related to possible insertions of external devices. Other causes of bruising, such as anticoagulant treatment or thrombocytopenia, should be considered carefully.

\section{INTERNAL EXAMINATION}

\section{Incisions}

The actual site and type of primary incision will depend on individual case circumstances and personal preference. A commonly recommended incision for the head and neck region begins behind the ears on both sides, passes down over the posterior-lateral aspect of the neck, and crosses the clavicles over the outer third. ${ }^{28}$ These two incisions are then joined in a curve meeting over the sternal angle. This combination exposes the neck structures and allows accurate assessment of the placement of any indwelling devices. The chest and abdominal incisions are usually in the midline anteriorly and care should be taken to dissect around inserted devices in order to allow accurate assessment of placement. Care must be taken to exclude surgical emphysema, pneumothorax, and air embolism at this early stage. Reflection of the skin and removal of the anterior rib cage before further dissection exposes all major organs and body cavities.

All effusions, haemorrhages, pus, and other fluid collections should be recorded and sampled at this stage. Some haemorrhage is often present at operative sites but more than $250 \mathrm{ml}$ is unusual. Blood and microbiological samples are also best taken before further dissection to avoid contamination from other sources. ${ }^{29}$ Blood cultures are best taken from the heart or spleen after searing the surface to eliminate contaminants. ${ }^{30}$ In cases involving known septicaemia, sepsis should be sought around the operative site, in the pelvis and subphrenic regions, and in the thorax after cardiothoracic surgery. Possible predisposing causes of septicaemia, such as malignancy, malnutrition, chronic alcoholism, and immunodeficiency should be excluded. Hospi- 
tal related infections should be discussed with the infection control team to inform them and to review the case within the overall context of the institution.

Extreme care should be used when examining the intestines in cases following abdominal surgery. Autolysis may complicate appearances, particularly in relation to recent suture lines at sites of anastamoses. Mere handling of tissues at necropsy may tear vital structures and prevent useful assessment. The presence of the surgeon can be invaluable in the accurate identification of local postoperative complications. Operative sites must always be examined in situ before the dissection, which may cause artefactual defects in organs and anastomoses.

Organ removal

The method of removal will vary according to the case and personal preference. ${ }^{28}$ If surgery has involved transdiaphragmatic structures such as the oesophagus, removal in one block is essential. ${ }^{31}$ This method also permits examination of the intact aorta, its major branches, and the major veins. Surgery involving the diaphragm itself is also best assessed using this method. Other methods may be useful in some circumstances and it is important that pathologists can adapt their dissection technique accordingly to get good in situ views of the site and system.

\section{Cardiovascular system}

DEMONSTRATION OF AIR EMBOLISM

Air embolism may occur in the arterial or venous systems. Venous air embolism may occur with open systems of intravenous infusion, secondary to trauma, and during parturition or abortion. ${ }^{32}$ Arterial air embolism may occur during cardiopulmonary bypass or chest surgery and with any trauma to the major pulmonary veins. The diagnosis of air embolism must be considered before necropsy because air may be introduced into vessels during dissection. ${ }^{33}$ Whole body radiography can be used to demonstrate air embolism. If air is seen in the heart or great vessels before dissection then it is not artefactual (provided there has not been significant putrefaction after death). Significant venous air embolism will fill the right atrium and ventricle with air. The pericardial sac must be exposed with minimum trauma to blood vessels. ${ }^{35}$ This is best achieved by removing the ribs up to the second rib and then dividing the sternum distal to the sternoclavicular joint, which allows the internal mammary vessels to be clamped. The pericardial sac is then filled with water and a water filled syringe, with plunger removed, is connected to a needle which is inserted into the right ventricle. Air bubbles appear if there has been a large venous air embolism. If air embolism is present then the inferior and superior vena cava should be clamped before removal of the heart so that the source of the embolism can be sought. The chest and abdominal cavities should be filled with water and the major veins tested using a similar technique to that used for the heart. A volume of $100-250 \mathrm{ml}$ of air is estimated to be required to cause death from venous air embolism, but much smaller amounts are required to cause death on the arterial side. The demonstration of arterial air embolism is therefore very difficult and extreme care must be taken during the removal of the brain (see central nervous system).

PERIOPERATIVE MYOCARDIAL INFARCTION

Perioperative myocardial infarction is uncommon in patients with no previous evidence of ischaemia but occurs in up to $5 \%$ of patients with ischaemic heart disease. ${ }^{36}$ The risk of infarction increases with age and severity of pre-existing cardiac failure. Perioperative infarcts are common after surgery for peripheral vascular disease. The majority of perioperative myocardial infarcts occur between one and three days after the surgical procedure, and clinical diagnosis can be extremely difficult. All evidence from preoperative assessments should be studied carefully, particularly ECGs. Careful macroscopic and histological examination of the myocardium is essential. Demonstration of myocardial infarction by enzyme techniques is time consuming and often inconclusive but newer techniques, such as C9 immunohistochemistry, may be more reliable. ${ }^{37}$ Serological measurement of cardiac enzymes may also be of some assistance. ${ }^{27}{ }^{39}$ Early macroscopic evidence of infarction is still easily overlooked and histology blocks should be taken from anterior, lateral, and posterior left ventricle, the interventricular septum, and the adjacent right ventricle. Evidence of chronic cardiac failure may be provided by histological changes of pulmonary hypertension, intra-alveolar haemosiderin deposition, and hepatic venous congestion.

\section{CARDIAC PACEMAKERS}

Cardiac pacing has become a major therapeutic tool in the treatment of a wide variety of conduction disturbances of the heart. Few deaths are directly related to the pacemaker or allied devices, but complications or failure may lead indirectly to a fatal outcome and pathologists must be familiar with such problems. ${ }^{40}$ Temporary pacemakers are commonly used in cardiac surgery patients to prevent arrhythmias or to support cardiac output.

Local complications may arise as a consequence of surgical implantation. These include haemorrhage, infection, and venous thrombosis. ${ }^{42}{ }^{43}$ Pacemaker or battery malfunction is rare but can easily be excluded with an appropriate testing facility, as found in most cardiothoracic units. Care must be taken not to allow the lead to come into contact with the pacemaker unit as this may cause artefactual circuit defects. Lead related problems include dislodgement, fractures, insulation breaks, and migration. Thick dense fibrous tissue may surround the endocardial electrode tip. ${ }^{43}$

CENTRAL VENOUS CATHETERS

Central venous catheters are widely used in the treatment of critically ill patients for the monitoring of central venous pressure and the administration of intravenous fluids, drugs, and parenteral nutrition. These devices are variable 
in design and may have single, double, or triple lumens. One of the commonest in use is the balloon tipped, flow directed pulmonary indwelling catheter (Swan-Ganz catheter). Complications with the catheter itself are not uncommon $^{44}$ but venous thrombosis and cardiac lesions are more common. Superficial haemorrhages may occur in the endocardium of both the right atrium and the right ventricle. Pulmonary valve petechial haemorrhage may also occur. The incidence of infections is low and is related to the duration of implantation. Endocardial and valvar damage by the catheter provides a nidus for the development of bacterial endocarditis. Skin commensals are the commonest pathogens and are best detected by cultures from sterile cardiac blood or from the catheter tip.

CARDIAC SURGERY RELATED DEATHS

General comments

Cardiac failure is the commonest cause of hospital mortality after both coronary artery bypass grafts and valve replacement. ${ }^{12} 153845$ Cardiac failure is a clinicopathological rather than purely necropsy derived diagnosis, defined as failure of the left ventricle resulting in pulmonary oedema. The diagnosis may be refined into preoperative and postoperative types. Lee and Gallagher ${ }^{45}$ define preoperative cardiac failure as the cause of death in patients who are receiving inotropes, have an intracardiac balloon pump, or have no spontaneous cardiac output when they go to theatre and have persistent cardiac failure after coming off cardiopulmonary bypass until death. They recognise perioperative or postoperative cardiac failure only if there are clinical features of cardiac failure developing during or after surgery and evidence of a new pathological change, such as myocardial infarction.

Transient postoperative arrhythmias occur in up to $25 \%$ of patients with previously normal rhythm after thoracic surgery. ${ }^{45}$ Age and obesity are contributory factors. Many unexplained deaths after cardiac surgery are spuriously attributed to ventricular arrhythmias. There is often no clinical evidence to support such diagnoses, and approximately $10 \%$ of deaths within 30 days of surgery remain unexplained in specialist cardiothoracic centres. Infection and neurological complications may occur but rarely contribute to death

Necropsies on patients who die after procedures requiring cardiopulmonary bypass present particular problems, and recent prothrombin times should be obtained by the pathologist. The pericardium is often firmly adherent to the external surface of the heart and care must be taken not to damage the myocardium or the sites of any surgery. Blood stained pericardial effusions are common in patients who have been actively resuscitated and these may be caused by attempts to aspirate pericardial contents, by intracardiac injections, or by rupture of epicardial vessels. The dissection of the heart will require modification according to individual surgical procedures, and usual techniques, such as following the flow of blood, are often inappropriate for clinicopathological correlation. Consideration should be given to dissecting the heart in echocardiographic planes. $^{35}$ Dissection after fixation is often useful and consideration should be given to referral to specialist cardiothoracic pathology centres in cases involving heart or lung transplantation.

\section{Coronary bypass grafts}

Coronary artery bypass grafting is now the commonest cardiac surgical procedure. The long term patency for saphenous vein grafts is approximately $60 \% .^{46}$ Better results are achieved with internal mammary, epigastric, and gastroepiploic artery grafts. Deaths following bypass procedures are usually related to myocardial infarction or pump failure, although no obvious cause is found in around $30 \%$ of cases. Haemorrhage is a major technical problem and all anastamoses must be inspected carefully. Grafts and coronary arteries should be sectioned transversely and histological examination should include anastamoses, proximal and distal coronary artery, and the left ventricular myocardium. Coronary artery angiography at necropsy, ${ }^{35}$ or even casts of the arteries, ${ }^{48}$ can be useful but these are time consuming activities and it may not be possible to incorporate them into routine necropsy practice. Acute infarcts following bypass surgery are almost all in the territory supplied by the vessel into which the graft has been inserted.

\section{Heart valve replacement}

A single transverse cut above the base of the heart will expose replacement aortic, mitral, and tricuspid valves. Important complications of valve replacement include infective endocarditis and perivalvar leaks. ${ }^{12} 4549-51$ True thrombus must be differentiated from postmortem clot which commonly forms around prosthetic valves. Structural failure is more common with porcine bioprosthetic valves than with mechanical valves. This is often secondary to calcification. In contrast, perivalvar regurgitation and haemorrhage related complications are more common with mechanical valves. Some specialist centres offer detailed assessments of replacement heart valve function and maximum information is obtained by analysis of unfixed valve rings

\section{Cardiac catheters and angioplasty}

The commonest complication associated with cardiac catheterisation is bleeding at the vascular access site in the groin. The risk of mortality is low at approximately $0.2 \%$. Cardiac arrhythmias, coronary artery dissection, cardiac perforation, and infection may occur ${ }^{52-57}$ and review of the angiography tapes with the cardiologist before necropsy is essential to identify areas of interest.

Percutaneous transluminal coronary artery angioplasty may produce damage around the margins of atheromatous plaques with consequential dissection. Other complications include thrombus formation, pericarditis, and distal embolisation of atheromatous debris. ${ }^{58} 59$ Fibrinous intimal proliferation produces re- 
stenosis in up to $30 \%$ of cases. ${ }^{6061}$ Immediate mortality is less than $1 \%$ but up to $4 \%$ of patients develop an acute myocardial infarct, while a similar proportion requires emergency coronary artery bypass grafting. New stenting techniques pose additional problems for the examining pathologist. ${ }^{62-64}$

VASCULAR SURGERY RELATED DEATHS

All vascular grafts should be examined carefully for placement and patency. Anastamoses must be visualised, and dissection is often complicated by extensive local haemorrhage. It may be necessary to expose the entire length of the vascular graft, and knowledge of the operative procedure is invaluable in this respect. Appropriate microbiological samples should be taken at the earliest opportunity before contamination from other sources. Accurate assessment of the degree of disease present in the natural vasculature is important, particularly in the locality of surgical anastamoses.

\section{Respiratory system}

GENERAL COMMENTS

Adhesions between the visceral and parietal pleura should be separated by blunt dissection. Efforts should be made to avoid tearing the lung parenchyma but occasionally the lungs may have to be cut from the chest wall. The parietal chest wall, including the ribs, should always be inspected for pleural plaques and tumours. The position of any chest drains should be noted. Appropriate precautions must be taken in all cases of suspected pulmonary tuberculosis.

The respiratory system is best examined after the cardiovascular system and oesophagus. Careful removal of the heart is essential and elevation of the base of the heart allows identification of individual pulmonary veins. Complete assessment of the pulmonary venous system requires the weight of the right ventricle-which does not normally exceed 65 $\mathrm{g}$ in adults - and the ratio of the weights of the right and left ventricles, normally between 2.3:1 and 3.3:1. This ratio is unaffected by fixation and is best determined using Foulton's method, in which the atria are removed by cutting around the atrioventricular ring. ${ }^{65}$ The right ventricle is removed by cutting along the margin of the interventricular septum, leaving the septum as part of the left ventricle. Fulton established criteria for normality whereby (a) the combined ventricular weight is less than $250 \mathrm{~g}$; (b) the free wall of the right ventricle (R) weighs less than $65 \mathrm{~g}$; (c) the left ventricle and septum together $(\mathrm{LV}+\mathrm{S})$ weigh less than $190 \mathrm{~g}$; and (d) the ratio LV+S:R lies between 2.3:1 and 3.3:1.

The lungs are best examined lobe by lobe for externally visible lesions and palpated for nodules and differences in texture. The pathologist must then decide whether distension is required for one or both lungs. In general, inflation is invaluable in the assessment of any diffuse lung disease, especially emphysema, and in the preservation of the airways. ${ }^{66}$ Incision and slicing followed by fixation is beneficial for optimising histological assessment of consoli- dated areas. Consultation with a specialist cardiothoracic centre may be useful in difficult cases. Routine histological blocks should include the apical segments of upper and lower lobes, basal segments, and major bronchus to include lymph nodes. Other blocks should be taken from macroscopically evident lesions. Some blocks should include pleura.

\section{POSTOPERATIVE PNEUMONIA}

Postoperative pneumonia occurs in approximately $20 \%$ of patients undergoing thoracic or abdominal surgery but is rare in other procedures in previously fit patients. ${ }^{6768}$ Chronic respiratory disease increases the incidence of postoperative pneumonia threefold. Other risk factors include obesity, age over 70 years, and operations lasting more than two hours. Samples taken under aseptic conditions at necropsy can be sent for microbiological culture. This improves the detection of postoperative pneumonia and enables organisms to be typed and tested for antibiotic sensitivity.

\section{POSTOPERATIVE THROMBOEMBOLISM}

The incidence of deaths from postoperative thromboembolism remains high (approximately $0.1 \%$ ) despite some reduction as a result of improvements in prophylactic measures and advances in anaesthesia and intensive care ${ }^{69-75}$ Risk factors include increased age, low mobility, malignancy, and vascular surgery. ${ }^{76-78}$ Detection of thromboembolism is facilitated by dissecting the heart in a manner that follows the blood flow. This permits careful inspection of the pulmonary trunk in continuity with the lobar branches, which can then be opened in sequence. If pulmonary thromboembolism is identified at necropsy the leg and pelvic veins should be examined for residual thrombus. This will usually involve serial slicing of the veins within the calf muscles using a posterior incision. The periprostatic plexus should be examined in males if no thrombus is detected in the leg veins. In cases involving massive thromboembolism there is often no detectable residual thrombus in the peripheral vessels.

DEMONSTRATION OF PNEUMOTHORAX

The possibility of a pneumothorax should always be considered before starting a necropsy examination, as procedures for demonstrating it must be performed before opening the thoracic cavity. The incidence of pneumothorax is increased in patients with bullous lung disease and in those who have been subjected to mechanical ventilation. ${ }^{79}$ Artefactual pneumothorax may also occur as the result of resuscitation procedures, and pathologists must interpret findings in the light of all clinical information. The simplest method of detection is to fill the space between the undamaged intercostal musculature and the reflected anterior skin with water; one of the intercostal spaces is then punctured with a scalpel and careful observation made for air escape. $^{35}$ 
Central nervous system

Many neurosurgical procedures are performed in cases involving trauma. A careful external examination should have identified any operation scars, contusions, or lacerations over the head and neck. Inequality in pupil sizes and conjunctival haemorrhages are also useful markers for intracranial pathology. The brain should always be removed by the pathologist and this enables accurate assessment of the distributions of haemorrhage and exudates. ${ }^{81}$ Cerebrospinal fluid and microbiological samples should be obtained at the earliest opportunity. If an encephalitis is suspected, representative samples of brain should be placed in appropriate transport media. Cerebrospinal fluid can be obtained by needle aspiration from the lateral ventricles or from the fourth ventricle by gentle compression of the brain. Spinal cerebrospinal fluid is obtained by tilting the head down after brain removal. Smear preparations can be used to provide a rapid diagnosis of neoplastic meningitis.

External examination of the brain often provides useful information; all abnormalities should be noted including contusions, haemorrhages, surface tumours, and atrophy, whether global or focal. Evidence of brain swelling should be sought, including flattening of cerebral gyri, uncal herniation, subfalcine herniation, and foraminal herniation. Many complex cases, for example those involving possible hypoxia or diffuse axonal injury, will require detailed internal inspection of the brain and histology. Referral of the intact fixed brain to a specialist centre is often worthwhile and most coroners are prepared to wait for a few weeks for a complete necropsy report including a neuropathological opinion.

If air embolism is suspected the cranial vault should be removed without puncturing the meninges. The meningeal arteries should be examined for bubbles with the brain in place. The internal carotid and basilar arteries must be clamped before transection of these vessels during removal of the brain from the cranial cavity. The inferior surface of the brain can then be immersed to facilitate detection of air bubbles within the vessels.

Ventricular shunts used for the treatment of obstructive hydrocephalus should be exposed in either the subcutaneous tissues or in the case of ventriculo-atrial shunts in the internal jugular vein. The tube should be opened and it is then possible to test the patency of the proximal and distal segments with a syringe and coloured dye.

\section{SPINAL CORD}

Examination of the spinal cord should be routine in any case involving a central nervous system disorder. Unsuspected spinal injury is a common finding in trauma cases and can be related to epidural or spinal anaesthesia. ${ }^{82}$ The spinal cord can be removed by anterior or posterior approaches, but the anterior approach provides less risk of cord damage and also facilitates sampling of dorsal root ganglia, nerve roots, and adjacent plexuses. Injuries to the craniocervical junction and high cervical spine, which may occur following minimal trauma during anaesthesia in patients with rheumatoid arthritis, are best demonstrated by removal of the entire cervical vertebral column. $^{83} 84$

\section{Hepatobiliary system}

Dissection of the hepatobiliary system is best performed from the posterior aspect. The portal venous system and splenic vein should be opened and examined for thrombi, leakage, and tumour. The common hepatic artery can be traced from the coeliac axis and this and the splenic artery should be examined for thrombi, leakage, and aneurysms. Dissection of the porta hepatis allows identification of the common bile duct which can be traced down to the ampulla and up into the hepatic ducts. A transverse cut into the tail of pancreas allows identification of the pancreatic duct and this can then be traced proximally. Any obstruction of the biliary or pancreatic duct systems is easily demonstrated, as is the positioning of stents and surgical clips following conventional or laparoscopic surgery.

\section{Gastrointestinal system}

A wide primary neck incision allows excellent exposure of the oropharyngeal structures, including salivary glands. Oesophageal pathology, such as varices and tumours of the gastrooesophageal junction, is best demonstrated by inversion of the oesophagus in direct continuity with the stomach.

The large and small intestines may be detached before removal of the main organ block, or removed in continuity with the organ block. ${ }^{85}$ The latter method is useful for ischaemic disease and allows for dissection of the intact vascular system. In cases of dense fibrous adhesions, the matted bowel loops may then be examined by making multiple slices, preferably in the coronal plane. Anastamoses require careful handling and assessment because postmortem autolysis can produce apparent perforations and dehiscence. Cases involving suspected perforated viscera may be similarly complicated by postmortem changes which increase with the interval between death and necropsy. Distension of the bowel with water under pressure to facilitate dissection is not advisable in such cases because of the risk of destruction of true pathology. Accurate identification of the source of gastrointestinal haemorrhage can be extremely difficult, particularly in the distal colon and rectum. Angiography can be useful in angiodysplasia but is only practicable in the intact bowel. Stomas are best removed in continuity with the intestines and this permits better appraisal of stoma related pathology such as stenosis, parastomal herniation, and inversion.

\section{Musculoskeletal system}

Most deaths following surgery on the skeletal system occur as a result of systemic complications such as septicaemia or thromboembolism. It is important to examine the site of surgery in order to exclude local complications of wound infection, haemorrhage, and displace- 
ment of inserted prostheses. The assessment of osteoporosis (or more accurately osteopenia) is important, since deaths resulting from fractures may be attributed to natural causes if severe osteoporosis is present. The traditional method of assessing osteopenia is to deform the cut end of a rib between thumb and forefinger until it fractures. Although ribs are mainly cortical bone, which is of less significance than medullary bone in osteoporotic fracture, this method is more reliable than the other method which involves assessments of the strength of hemivertebral bodies by direct pressure after removal of the anterior aspects. These simple subjective assessments of bone fracturability are acceptable and cost-effective measures of osteoporosis. ${ }^{86}$ Bone histomorphometry often provides no additional information.

Samples of bone marrow can provide useful information in some cases, particularly those involving known or suspected haematological malignancies. Small samples should be taken from at least three separate sites and can be processed in a similar way to diagnostic bone marrow trephines. Reasonable morphology can be achieved but this depends on the sampling technique and the interval between death and necropsy.

\section{Head and neck region}

Necropsy technique must be modified according to the site and nature of the surgical procedure. ${ }^{87}$ The lower cranial nerves are best examined after removal of the sternomastoid muscle. The cervical sympathetic chain is found in the prevertebral connective tissue and should be sampled with the vagus nerve in cases of possible autonomic failure. ${ }^{88}$

Haemorrhage within the anterior neck structures may result from surgery, intubation, or insertion of central venous catheters for a variety of purposes. All vascular structures should be opened with the heart and aortic arch intact. ${ }^{89}$ This facilitates identification and demonstration of the placement of indwelling devices. All cases involving suspected cerebrovascular disease will require a detailed examination of the extracranial cerebral arteries. It may be necessary to examine the vertebral arteries if there is a history of vertebrobasilar insufficiency, and a simple method by which these vessels can be dissected out of the transverse processes at necropsy has been described. ${ }^{90}$

The thyroid gland is best removed whole, although for accurate assessments of the extent of invasion of thyroid and laryngeal tumours, removal of the neck block intact is useful. This can then be fixed and prepared in a similar way to laryngectomy specimens. Assessment of specialised ophthalmic and maxillofacial surgical procedures requires close liaison with the relevant clinicians before necropsy. Consideration must be given to the need for disfiguring dissection which will not provide any additional information about the cause of death.
Important concepts in necropsy of anaesthetic related deaths

Morphological findings may be minimal or absent

Toxicological investigations are often inconclusive

Local, epidural and general anaesthesia have different associated complications

Anaesthesia may contribute to deaths that occur more than 24 hours after administration

Rapid developments in anaesthetic technology result in use of complex anaesthetic agents and devices

Pathologists need expert independent advice on anaesthetic techniques and agents

Deaths associated with anaesthesia

GENERAL COMMENTS

Any necropsy arising from a known or suspected anaesthetic death will require consultation with the anaesthetist. Morphological findings may be minimal or absent, and numerous anaesthetic devices may have been introduced into the patient during the procedure or subsequently. Anaesthesia may also contribute to deaths that occur more than 24 hours after administration. Most pathologists do not have the necessary training or experience for a critical assessment of the many applications of complex modern anaesthesia without expert assistance, and consideration should always be given to independent sources of advice.

CAUSES OF DEATH IN GENERAL ANAESTHESIA

Most anaesthetic related deaths are not caused by the anaesthetic agent itself but by other aspects of the procedure or by serious intercurrent disease. Major contributory factors in many true anaesthetic fatalities are lack of experience and supervision among junior medical staff, particularly when these allow periods of hypoxia to occur.

The commonest cause of death is cardiac arrest which often occurs either at the start or at the finish of the surgical procedure. The pathogenesis of the cardiac arrest is often neurogenic and can be caused by any irritation of the respiratory tract, such as intubation or laryngoscopy, in the lightly anaesthetised patient. Surgical manipulation may cause cardiac arrest by a similar mechanism. Other causes of cardiac arrest include decreased myocardial perfusion secondary to lowering of the diastolic blood pressure or heart rate during induction, particularly in patients with pre-existing coronary artery disease.

Hypoxia may also precipitate cardiac arrest, and all possible causes of respiratory failure
Considerations in anaesthetic related deaths
Endotracheal tubes
Vascular cannulas
Aspiration of gastric contents
Epidural/spinal anaesthesia
Malignant hyperthermia
Halothane hepatitis 
must be evaluated carefully, particularly when there is known intercurrent respiratory disease. Inexperience or unfamiliarity with anaesthetic apparatus may result in overdose of anaesthetic agent, causing depression of respiratory centres and hypoxia. Excessive premedication and the use of muscle paralysing agents may also predispose to respiratory failure without appropriate assistance with respiration. Airway obstruction is another danger and may result from blood, teeth, dentures, swabs, laryngeal spasm, abnormal neck posture, and faults in apparatus. Regurgitation of gastric contents may occur but this is uncommon because of the general use of cuffed endotracheal tubes. The presence of gastric contents in airways must be confirmed by additional clinical evidence, as such findings are present in approximately $25 \%$ of all deaths. Movement of the body and agonal events are the likely mechanisms in most cases.

Physical faults in anaesthetic equipment rarely cause death. When failure of apparatus is possible, then expert examination and advice is mandatory. Anaesthetic machines, gas supply, connecting tubes, and all other hardware must be subjected to rigorous examination if malfunction is suspected. Pathologists must ensure that they receive any conclusions relating to possible equipment failure, as these may influence the eventual decision about the cause of death. Incompatibility within connecting tubing can cause internal delamination and luminal occlusion. Flowmeter errors, use of wrong piped or bottled gas supply, and connection of empty cylinders are all possible.

\section{NECROPSY PROCEDURE IN ANAESTHESIA RELATED} DEATHS

The main function of necropsy in these cases is to confirm or exclude natural disease. The pharmacological aspects of anaesthetic related deaths are often impossible to investigate in any detail. Toxicological investigations are usually unhelpful except in cases involving overdose with specific drugs such as barbiturates or adrenaline (epinephrine). It is impossible to make any reliable quantitative assessments of any volatile agents present at necropsy and similarly blood gas measurements cannot confirm the presence of hypoxia. Antemortem blood samples should be retrieved and analysed in cases of suspected malignant hyperpyrexia.

Numerous anaesthetic devices may have been introduced into the patient during treatment, such as endotracheal tubes, indwelling catheters, intravascular cannulas, and monitoring electrodes. All such items must be left in place after death to allow assessments of placement and patency. If clinical data raise doubt about placement, consideration should be given to pre-necropsy radiology to identify exact anatomical positioning. Oesophageal intubation is uncommon but can be confirmed by using a primary midline neck incision. If the position has been rectified after death, then a ring of oedematous oesophageal mucosa may be visible at the level of the inflatable component. Distension of the stomach and intestines may occur with use of anaesthetic gas in such cases but analysis for the agent is usually unsuccessful.

A full range of histology should be taken not only to exclude occult intercurrent disease but also to assess the severity and extent of the disease for which the surgery was necessary. Histology can also confirm specific anaesthetic complications such as halothane hepatitis. Retention and examination of the intact brain for detailed neuropathological assessment is essential but many deaths will occur before the development of changes associated with hypoxia.

LOCAL AND EPIDURAL ANAESTHESIA

Local anaesthetics rarely cause death. Hypersensitivity and escape of contained adrenergic drugs with resulting overdose are the major hazards. Diffusion away from the injection site may cause vasoconstriction and cardiac arrest. ${ }^{91}$ The upward diffusion of active agents in spinal anaesthesia may produce fatal hypotension through involvement of the vasomotor system. If concentrations of spinal fluid anaesthesia in the upper cervical and thoracic regions are high enough, efferent transmission to all respiratory muscle motor fibres will be blocked, leading to respiratory arrest. Problems in epidural anaesthesia are rare and usually arise from overdose and thecal puncture. Necropsy samples of cerebrospinal fluid should be analysed for concentrations of anaesthetic substances. The location and patency of epidural catheters can be assessed by the introduction of a dye and careful subsequent dissection.

MALIGNANT HYPERTHERMIA

This is an autosomal dominant condition in which certain agents, including some anaesthetics, precipitate a metabolic change in skeletal muscle with the production of energy and a rapid rise in temperature. ${ }^{92-94}$ Succinylcholine and halogenated anaesthetic agents such as halothane may precipitate the condition, which occurs in about one in 10000 anaesthetics. There are no necropsy findings of note and diagnosis depends on clinical evidence. In $70 \%$ of carriers, the creatine phosphokinase and aldolase activities are raised and the diagnosis can be confirmed by analysis of antemortem blood. Other family members should be screened for presence of the disease. Other anaesthetic agents have particular complications associated with their administration. Halothane anaesthesia may cause hepatitis after repeated use. An excess of barbiturates used in induction, such as thiopentone (thiopental), may produce cardiopulmonary arrest.

\section{Recording necropsy findings}

The use of body charts is recommended for recording all macroscopic features such as body height, body weight, placement sites of external and internal devices, fracture sites, surgical scars, evidence of trauma, and organ weights. These proforma charts may also serve as histology request forms for laboratory use and can be retained permanently unlike 
cassette tapes which are used repeatedly. Conventional photography remains more accessible and useful than still video or digital imaging but neither must be regarded as a replacement for detailed descriptions of relevant necropsy findings. ${ }^{95}$ There may also be medicolegal restrictions on the retention of all such images.

\section{Further investigations}

Histology from specialised sites may be required in certain types of necropsy but it is a useful routine to retain a standard set of tissues from each of the major organs.

It is often not possible to obtain more tissue at a later date and samples of other tissues should be retained if they might possibly contribute to establishing the cause of death. In the United Kingdom neither the coroner nor the procurator fiscal can authorise the retention of blood or tissue for research. The consent, or at least absence of objection, from the relatives of the deceased must be obtained, together with the consent of the appropriate medicolegal authority to tissue retention in any necropsy carried out under their jurisdiction. Pathologists should be aware of the legal restrictions which limit the retention of material from medicolegal necropsies. Pathologists must also be familiar with the techniques required to obtain appropriate microbiological and toxicological samples. ${ }^{30}{ }^{96}$ Extensive guidelines are available and any uncertainty should be clarified before necropsy. Sepsis with multiorgan failure is a major cause of postoperative mortality.

\section{Communication with clinicians}

Clinicopathological communication is essential if the maximum possible information is to be obtained from necropsies. This process begins before the necropsy examination with review of the clinical notes. It is often useful to request that the clinicians summarise the clinical history and state the questions to be answered by the necropsy. Some cases may require the presence of the surgeon or other relevant clinician during the examination, and with sensible liaison a mutually acceptable time to perform the necropsy can usually be found. Discussion in the mortuary during demonstration of the gross necropsy findings is also essential and may identify further areas for examination. All necropsies should be discussed at appropriate regular clinicopathological meetings, where the gross findings can be supplemented by appropriate histological, microbiological, and toxicological investigations. Such presentations are enhanced by the use of conventional photography to record interesting findings. Consideration should also be given to regular mortality meetings as part of the clinical audit process. Many centres now routinely record data relating to discrepancies between clinical diagnoses and necropsy findings. This is worthy of consideration and acceptable classifications exist for such purposes. ${ }^{97}$ Pathologists should be as supportive of their clinical colleagues as objective evaluation circumstances allow, and they should not precipitate unnecessary medicolegal or disciplinary proceedings by hasty release of preliminary findings or ill founded speculation. Dissecting the abdominal contents at necropsy of a subject who died from a ruptured abdominal aortic aneurysm is completely different from trying to repair it during life and pathologists sometimes forget the pressures that clinicians work under. Unless there are obvious gross deficiencies in clinical performance, in which case the appropriate medicolegal authority should be contacted independently of the clinicians, the pathologist should present the findings in a clear objective manner with no judgmental implications.

\section{Necropsy reports}

National guidelines for necropsy reports have been produced and these include standards for content and timeliness. ${ }^{98}$ Actual content will vary according to the nature of the individual case, and specialised reports may be appropriate, depending on the type of surgical procedure performed. However, all reports should contain three partitions of information: (1) actual findings at necropsy; (2) explanation of these findings and conclusions; (3) the cause of death in a format appropriate to the medicolegal system

The actual findings are the result of expert skills in the performance of necropsies, but the pathologist should regard these as a record witnessing the "facts" of the case in a similar manner to a statement given to police after witnessing a road traffic accident or burglary.

Special features in necropsies on postoperative deaths

(1) Cardiovascular system

(a) Air embolism

(b) Perioperative myocardial infarction

(c) Cardiac pacemaker

(d) Central venous catheters

(e) Cardiac surgery

(i) Coronary bypass grafts

(ii) Heart valve replacement

(2) Respiratory system

(a) Postoperative pneumonia

(iii) Angioplasty

(b) Thromboembolism

(c) Pneumothorax

(3) Central nervous system

(a) Air embolism

(b) Ventricular shunts

(4) Hepatobiliary system

(5) Gastrointestinal system

(a) Stents and clips

(6) Musculoskeletal system

(a) Anastomoses

(a) Prostheses 
General checklist for performing a necropsy examination on a postoperative death

(1) Review of case

(2) Consideration of medicolegal status

(3) Necropsy dissection

(a) External examination

(i) Operation incisions

(ii) Drain sites

(iii) Vascular cannulae

(iv) Endotracheal tube

(v) Urinary catheter

(b) Internal examination

(i) Standard dissection modified by circumstances (see table 6)

(ii) Sampling for microbiology, toxicology and histology as appropriate

(4) Necropsy report

(i) Factual findings

(ii) Explanations and opinions

(iii) Cause of death

Of course some perception or interpretation is required to identify these facts but this section should stop at that point and not offer explanation or opinion about the observations. Examples of such records of fact could be "There was a metal clip, $5 \mathrm{~mm}$ in length, applied transversely to the external surface of the common bile duct which was compressing it with occlusion of the lumen" or "There was a $10 \mathrm{~mm}$ diameter deficit in the wall of the colon adjacent to the stapled anastomosis. The tissue at the edges of the deficit appeared necrotic and the adjacent bowel had a dusky appearance with a matt serosal surface."

The explanation of the actual findings and reasoned conclusions constitute expert evidence and form the area most likely to be challenged by other experts in any subsequent legal proceedings. The pathologist should devote much time and attention to this section in order to avoid protracted questioning and wrangling over loosely worded phrases. $\mathrm{Pa}-$ thologists should never substitute their "amateur" opinion on a specialist matter for that of a recognised specialist in the field. Although pathologists will have had some clinical experience before entering laboratory medicine, this experience will be limited and at least five years out of date when they are writing such necropsy reports, so they should make only the barest of statements about clinical matters and then defer to more specialist opinion. In the bile duct example in the previous paragraph the conclusions might be "Application of clips to the common bile duct is not a recognised part of the procedure of laparoscopic cholecystectomy, raising the question of standards of clinical performance, and this should be considered or investigated by an independent specialist in that field." However, the pathologist is an expert in pathology and can use this knowledge to provide helpful conclusions about the contribution of natural disease to the deceased's demise. In the colon example from the preceding paragraph the conclusions might include "This patient was a known arteriopath with severe atherosclerosis of the abdominal aorta and its branches. Two years prior to death he had an above knee amputation of the right leg for ischaemia due to peripheral vascular disease. The patient was a poor anaesthetic risk but the operation to remove the obstructing carcinoma of the sigmoid colon was required as a life saving procedure. At necropsy there was severe atherosclerosis of all abdominal branches of the aorta including the mesenteric arteries. The deficit in the bowel had necrotic edges and the colon adjacent to the deficit showed ischaemic changes. The balance of probabilities is strongly in favour of the deficit arising in the postoperative period secondary to ischaemia of the bowel due to the patient's severe atherosclerotic disease rather than an intraoperative event." However, some pathologists would regard these conclusions as overinterpretative and would just state the possibilities that could have caused the deficit with some weighting between them.

The statement of the "cause of death" is an area that is fraught with difficulty. ${ }^{99}{ }^{100}$ In Britain an epidemiologically oriented format is used which could be helpful for collecting data on the cause of death (if this was validated by necropsy examination in the majority of cases) but has great problems in the medicolegal context. The British format gives the cause of death in this format:

Ia - Disease or condition directly leading to death.

Ib - Other disease or condition, if any, leading to Ia.

Ic - Other disease or condition, if any, leading to Ia.

II - Other significant conditions contributing to the death but not related to the disease or condition causing it.

The main problem is that the cause of death provides a three or four line "summary" of the case which lawyers and medicolegal authorities seize upon as a categorical certainty, when in reality it is a statement of balance of probabilities constrained by an unhelpful format. The division of pathologies between part I and part II is largely a question of arbitrary semantics and the subdivision of part I gives the impression of a single causal chain of events which mitigates against a multifactorial explanation. Certainly true elements can be stated in the cause of death but uncertain elements are better left in the explanations and opinions section. If the case cannot be adequately fitted within the constraints of the cause of death then terms such as "undetermined" or "multiple factors" (see discussion above) can be used and the final wording can be left to the appropriate medicolegal authority, such as the coroner in England and Wales.

Many pathologists have problems in determining a cause of death in postsurgical deaths. The central issue is whether death would have occurred when it did if the operation had not taken place. In some cases this may be impossible to determine and any uncertainty should be made clear in the necropsy report. This often arises when both trauma and surgery have taken place, for it can be difficult to separate the relative contributions of each to a 
death. Where death is caused by a disease other than that for which the procedure was performed, a distinction must be drawn between those conditions that were known before the procedure and those which were unsuspected. When disease is known to be present, this must be evaluated to determine whether there was justification for the procedure. Unsuspected disease is only a problem if no reasonable precautions were taken to detect common risk factors. Exceptionally, death may result from a failure of anaesthetic or surgical technique. This may be inadvertent or a true accident which may arise from unusually difficult operative circumstances, from abnormal anatomy, or from equipment failure. If there is potential individual error or incompetence, then legal action for negligence may ensue and the pathologist must be meticulous in producing a detailed, objective, and impartial necropsy report.

The necropsy report must satisfy the needs of different recipients which may include the medicolegal authority (HM Coroner in England and Wales), the clinicians, the deceased's family doctor, relatives of the deceased, hospital managers, medical insurers, and lawyers acting for many different parties. It is impossible to write a fully comprehensive report using a vocabulary that could be understood by all these people, but the report should be written as clearly as possible given the need for a full technical content.

Access to necropsy reports is a controversial issue and is subject to the control of the local coroner or equivalent medicolegal authority. Local guidelines are essential, as necropsies associated with surgical procedures and anaesthesia will inevitably raise difficult issues regarding confidentiality and disclosure. $\mathrm{Pa}-$ thologists and clinicians must develop close working relationships with local coroners, and appropriate medicolegal education is invaluable.

We thank Prof A R W Forrest and Dr C Gray for their helpful comments on this manuscript.

1 Knight B. Deaths associated with surgical procedures. In: Knight B, ed. Forensic pathology. London: Arnold, 1996:475-81.

2 Fernandez-Segoviano P, Lazaro A, Esteban A, et al. Autopsy Fernandez-Segoviano P, Lazaro A, Esteban A, et al. Autopsy
as quality assurance in the intensive care unit. Crit Care as quality assurance

3 Hill RB, Anderson RE. The autopsy and health statistics. Leg Med 1990;5:57-69.

4 Shanks JH, McCluggage G, Anderson NH, et al. Value of the necropsy in perioperative deaths. F Clin Pathol 1990;43 193-5.

5 Stothert JC, Gbaanador GB, Herndon DN. The role of autopsy in death resulting from trauma. F Trauma 1990;30: 1021-5.

6 The Royal College of Pathologists. The autopsy and audit. London: The Royal College of Pathologists, 1991.

7 Shanks JH, Anderson NH, McCluggage G, et al. Use of the autopsy in Northern Ireland and its value in perioperative deaths. Lyon: IARC Scientific Publications, 1991:115-24.

8 Gatzoulis MA, Sheppard MN, Ho SY. Value and impact of necropsy in paediatric cardiology. Heart 1996;75:626-31.

9 Boldy DA, Jones C, Matthews H, et al. Deaths and necropsies in a thoracic unit. Thorax 1993;48:284-6.

10 Champ CS. Surgical audit without autopsy: tales of the unexpected. Ann R Coll Surg Engl 1993;75:302-3.

11 Mosquera DA, Goldman MD. Surgical audit without autopsy: tales of the unexpected. Ann R Coll Surg Engl 1993;75:115-17.

12 Lee AS, Borek BT, Gallagher PJ, et al. Prospective study of the value of necropsy examination in early death after cardiac surgery. Heart 1997;78:34-8.
13 Smith CJ, Scott SM, Wagner BM. The necessary role of the autopsy in cardiovascular epidemiology. Hum Pathol 1998; 29:1469-79.

14 Dearborn JT, Harris WH. Postoperative mortality after total hip arthroplasty - an analysis of deaths after two thousand seven hundred and thirty-six procedures. F Bone foint Surg Am 1998;18:1291-4

15 Deiwick M, Lohrer A, Hoffmeier A, et al. Postoperative death should be followed by autopsy-an analysis of the autopsy findings of the years 1990 and 1991 in a heart surgery center. Thorac Cardiovasc Surg 1999;47:82-7.

16 Mort TC, Yeston NS. The relationship of pre mortem diagnoses and post mortem findings in a surgical intensive care unit. Crit Care Med 1999;27:299-303.

17 Campling EA, Devlin HB, Lunn JN. The report of the National Confidential Enquiry into Perioperative Deaths 1989. London: HMSO, 1990.

18 Campling EA, Devlin HB, Hoile RW, et al. The report of the National Confidential Enquiry into Perioperative Deaths 1990. London: HMSO, 1992 .

19 Campling EA, Devlin HB, Hoile RW, et al. The report of the National Confidential Enquiry into Perioperative Deaths 1991/ 1992. London: HMSO, 1993.

20 Campling EA, Devlin HB, Hoile RW, et al. The report of the National Confidential Enquiry into Perioperative Deaths 1992/ 1993. London: HMSO, 1995

21 Cross SS. Autopsies, the deceased's relatives and the law. In: Cotton DWK, Cross SS, eds. The hospital autopsy. Oxford: Butterworth-Heinemann; 1993:8-14.

22 Human Tissue Act 1961.

23 Hudson M. Right of possession in human corpses. 7 Clin Pathol 1997;50:90-1.

24 Start RD, Delargy-Aziz Y, Dorries CP, et al. Clinicians and the coronial system: ability of clinicians to recognise reportable deaths. BMF 1993;306:1038-41.

25 Start RD, Usherwood TP, Carter N, et al. General practitioners' knowledge of when to refer deaths to a coropractitioners' knowledge of when to

26 Cox DE, Sadler DW, Pounder DJ. Alcohol estimation at necropsy: epidemiology, economics, and the elderly. $f$ Clin Pathol 1997;50:197-201.

27 Osuna E, PerezCarceles MD, Vieira DN, et al. Distribution of biochemical markers in biologic fluids-application to the postmortem diagnosis of myocardial infarction. $A m \mathcal{F}$ Forensic Med Pathol 1998;19:123-8.

28 Cotton DWK. The basic hospital autopsy. In: Cotton DWK, Cross SS, eds.The hospital autopsy, 1st ed. Oxford: Butterworth Heinemann, 1993:37-66.

29 Hove M, Pencil SD. Effect of postmortem sampling technique on the clinical significance of autopsy blood cultures. Hum Pathol 1998;29:137-9.

30 Spencer RS. The microbiology of the autopsy. In: Cotton DWK, Cross SS, eds. The hospital autopsy, 1st ed. Oxford: Butterworth Heinemann, 1993:144-57.

31 Culora GA, Roche WR. Simple method for necropsy dissection of the abdominal organs after abdominal surgery. F Clin Pathol 1996;49:776-9.

32 Saad RG. Fatal air embolism: a complication of manipulation of a cavitating metastatic lesion of the liver. Eur $\mathcal{F}$ Anaesthesiol 1998;15:372-5.

33 Bajanowski T, Kohler H, DuChesne A, et al. Proof of air embolism after exhumation. Int $\mathcal{F}$ Legal Med 1998;112:2-7.

34 Bajanowski T, West A, Brinkmann B. Proof of fatal air embolism. Int f Legal Med 1998;111:208-11.

35 Cotton DWK, Cross SS. Special procedures. In: Cotton DWK, Cross SS, eds. The hospital autopsy. Oxford: Butterworth-Heinemann, 1993:67-87.

36 Dawood MM, Gutpa DK, Southern J, et al. Pathology of fatal perioperative myocardial infarction: implications regarding pathophysiology and prevention. Int 7 Cardiol 996;57:37-44.

37 Doran JP, Howie AJ, Townend JN, et al. Detection of myocardial infarction by immunohistological staining for $\mathrm{C} 9$ on formalin fixed, paraffin wax embedded sections. F Clin Pathol 1996;49:34-7.

38 Hansen SH, Rossen K. Evaluation of cardiac troponin I immunoreaction in autopsy hearts: a possible marker of early myocardial infarction. Forensic Sci Int 1999;99:18996.

39 Osuna E, PerezCarceles MD, Alvarez MV, et al. Cardiac troponin I (cTn I) and the postmortem diagnosis of myocardial infarction. Int $\mathcal{f}$ Legal Med 1998;111:173-6.

40 Prahlow JA, Guileyardo JM, Barnard JJ. The implantable cardioverter-defibrillator. A potential hazard for autopsy cardioverter-deibrillator. A potential hazard for auto
pathologists. Arch Pathol Lab Med 1998;121:1076-80.

41 Laguno M, Miro O, Font C, et al. Pacemaker-related endocarditis - report of 7 cases and review of the literature. Cardiology 1998;90:244-8.

42 Cacoub P, Leprince P, Nataf P, et al. Pacemaker infective endocarditis. Am f Cardiol 1998; 82:480-4.

3 Candinas R, Duru F, Schneider J, et al. Postmortem analysis of encapsulation around long-term ventricular endocardial pacing leads. Mayo Clin Proc 1999;74:120-5.

44 O'Farrell L, Griffith JW, Lang CM. Histologic development of the sheath that forms around long-term implanted central venous catheters. $\mathcal{F}$ Parenter Enter Nutr 1996;20:156-8.

45 Lee AS, Gallagher PJ. Post-mortem examination after cardiac surgery. Histopathology 1998;33:399-405. 46 Davies MJ. Coronary artery remodelling and the assessment
of stenosis by pathologists. Histopathology 1998;33:497500 .

47 Gonschior P, Milz S, Lehr HA. Thrombocyte adhesion and leukocyte inflammation after angioplasty: a study of autopsy vessels. Circulation 1998;98:102. 
48 Weman SM, Salminen US, Penttila A, et al. Post-mortem cast angiography in the diagnostics of graft complications
in patients with fatal outcome following coronary artery in patients with fatal outcome following coronary artery
bypass grafting (CABG). Int $\mathcal{f}$ Legal Med 1999;112:107bypass

49 Schoen FJ, Titus JL, Lawrie GM. Autopsy-determined causes of death after cardiac valve replacement. $7 A M A$ 1983;249:899-902.

50 Ishibashi-Ueda $\mathrm{H}$, Imakita $\mathrm{M}$, Katsuragi $\mathrm{M}$, et al. An analysis of autopsy findings in 108 patients who died after valve replacement. Virchows Arch A Pathol Anat Histopathol 1993;422:397-403

51 Burke AP, Farb A, Sessums L, et al. Causes of sudden cardiac death in patients with replacement valves: an autopsy study. F Heart Valve Dis 1994;3:10-16.

52 Murray BH, Cohle SD, Davison P. Pericardial tamponade and death from Hickman catheter perforation. Am Surg 1996;62:994-7.

53 Collier PE, Blocker SH, Graff DM, et al. Cardiac tamponade from central venous catheters. Am $\mathcal{F}$ Surg 1998 176:212-14.

54 Timsit JF, Farkas JC, Boyer JM, et al. Central vein catheterrelated thrombosis in intensive care patients-incidence, risks factors, and relationship with catheter-related sepsis. Chest 1998;114:207-13.

55 Lamas CC, Eykyn SJ. Hospital acquired native valve endocarditis: analysis of 22 cases presenting over 11 years. Heart 1998;79:442-7.

56 Ramnath N, Momin F, Karanes C, et al. Fatal mucormycosis after intravascular catheterization in a transplant recipient. Infect Med 1998;15:252-5.

57 Papaleo R, Depowski PL, Dincer AD, et al. Left ventricular free wall rupture during coronary intervention after acute myocardial infarction: report of two cases exhibiting fatal pseudocomplications. Cathet Cardiovasc Intervent 1999; 47:67-72.

58 Bowles M, Palko W, Beaver C, et al. Clinical and postmortem outcome of 'no-reflow' phenomenon in a postmortem outcome of "no-reflow phenomenon in a patient treated

59 Veijola M, Ikaheimo M, Valkama J, et al. Fatal occlusion of the left coronary main stem by a fragment from the femoral artery: a previously unreported complication of cardiac catheterization. Forensic Sci Int 1997;88:111-16.

60 Waller BF, Fry EA, Peters TF, et al. Abrupt ( $<1$ day), acute $(<1$ week), and early $(<1$ month) vessel closure at the angioplasty site. Morphologic observations and causes of closure in 130 necropsy patients undergoing coronary angioplasty. Clin Cardiol 1996;19:857-68

61 Nakamura Y, Zhao H, Yutani C, et al. Morphometric and histologic assessment of remodeling associated with restenosis after percutaneous transluminal coronary angioplasty. Cardiology 1998;90:115-21.

62 Madan M, Lemay MR, Walley VM, et al. Coronary stenting in acute myocardial infarction-patency demonstrated in acute myocardial infarction-patency

63 Malik N, Gunn J, Holt CM, et al. Intravascular stents: a new technique for tissue processing for histology, immunohistochemistry, and transmission electron microscopy. Heart chemistry, and

64 Farb A, Lindsay J, Virmani R. Pathology of bailout coronary stenting in human beings. Am Heart f 1999;137:621-31.

65 Foulton RM, Hutchinson EC, Jones AM. Ventricular weights in cardiac hypertrophy. Br Heart f 1952;14:41320.

66 McCulloch TA, Rutty GN. Postmortem examination of the lungs: a preservation technique for opening the bronch and pulmonary arteries individually without transection problems. F Clin Pathol 1998;51:163-4

67 Kirtland SH, Corley DE, Winterbauer RH, et al. The diagnosis of ventilator-associated pneumonia - a comparison of histologic, 1997;112:445-57

68 Yaffe MB, Fink MP. Hospital-acquired pneumonia in the postoperative setting. Semin Resp Crit Care Med 1997;18: 121-32.

69 Macintyre IM, Ruckley CV. Pulmonary embolism: a clinical and autopsy study. Scot Med $\mathcal{7}$ 1974;19:20-4.

70 Bergqvist D, Lindblad B. A 30-year survey of pulmonary embolism verified at autopsy: an analysis of 1274 surgical patients. Br f Surg 1985;72:105-8.

71 Rubinstein I, Murray D, Hoffstein V. Fatal pulmonary emboli in hospitalized patients: an autopsy study. Arch Intern Med 1988;148:1425-6.
72 Sandler DA, Martin JF. Autopsy proven pulmonary embolism in hospital patients: are we detecting enough deep vein thrombosis? $\mathcal{F} R$ Soc Med 1989;82:203-5.

73 Lindblad B, Eriksson A, Bergqvist D. Autopsy-verified pulmonary embolism in a surgical department: analysis of the period from 1951 to 1988. Br F Surg 1991;78:849-52.

74 Karwinski B, Svendsen E, Seim S. Pulmonary embolism and heart disease: an autopsy study. Pathol Res Pract 1993; 189:1058-62.

75 Scurr J, Baglin T, Burns H, et al. Risk of and prophylaxis for venous thromboembolism in hospital patients. Phlebology 1998;13:87-97.

76 Derish MT, Smith DW, Frankel LR. Venous catheter thrombus formation and pulmonary embolism in children. Pediatr Pulmonol 1995;20:349-54.

77 Cohen AT, Edmondson RA, Phillips MJ, et al. The changing pattern of venous thromboembolic disease. Haemostasis 1996;26:65-71.

78 Eriksson S, Backman L, Ljungstrom KG. The incidence of clinical postoperative thrombosis after gastric surgery for obesity during 16 years. Obesity Surg 1997;7:332-5.

79 Klein JS, Zarka MA. Transthoracic needle biopsy: an overview. F Thorac Imaging 1997;12:232-49.

80 Sharma KC, Kabinoff G, Ducheine Y, et al. Laparoscopic surgery and its potential for medical complications. Heart Lung 1997;26:52-64.

81 Powers JM. Practice guidelines for autopsy pathology. Autopsy procedures for brain, spinal cord, and neuromuscular system. Autopsy Committee of the College of American Pathologists. Arch Pathol Lab Med 1995;119:777-83.

82 Greaves JD. Serious spinal cord injury due to haematomyelia caused by spinal anaesthesia in a patient treated with low-dose heparin. Anaesthesia 1997;52:150-4.

83 Geddes JF, Gonzalez AG. Examination of spinal cord in diseases of the craniocervical junction and high cervical spine. 7 Clin Pathol 1991;44:170-2.

84 Berzlanovich AM, Sim E, Muhm MA. Technique for dissecting the cervical vertebral column. I Forensic Sci 998;43:190-3.

85 Barendregt WB, de Boer HH, Kubat $\mathrm{K}$. The results of autopsy of patients with surgical diseases of the digestive tract. Surg Gynecol Obstet 1992;175:227-32.

86 Harris SC, Cotton DWK, Stephenson TJ, et al. Assessment of osteopenia at autopsy. Med Sci Law 1991;31:15-18.

87 Bagatella F. Technique for removal of the nasosinusal block at autopsy. Rhinology 1981;19:47-50.

88 Adams VI. Autopsy technique for neck examination. II. Vertebral column and posterior compartment. Pathol Annu 1991;26:211-26.

89 Adams VI. Autopsy technique for neck examination. I. Anterior and lateral compartments and tongue. Pathol Аппи 1990;25 Pt 2:331-49.

90 Bromilow A, Burns J. Technique for removal of vertebral arteries. F Clin Pathol 1985;38:1400-2.

91 Sprung J, Lesitsky MA, Jagetia A, et al. Cardiac arrest caused by coronary spasm in two patients during recovery from epidural anesthesia. Reg Anesth 1996;21:253-60.

92 BenAbraham R, Cahana A, KrivosicHorber RM, et al. Malignant hyperthermia susceptibility: anaesthetic implications and risk stratification. F Assoc Physicians 1997;90: 13-18.

93 Loke J, MacLennan DH. Malignant hyperthermia and central core disease: disorders of $\mathrm{Ca}^{2+}$ release channels. $\mathrm{Am} \mathcal{F}$ Med 1998;104:470-86.

94 BenAbraham R, Adnet P, Glauber V, et al. Malignant hyperthermia. Postgrad Med f 1998;74:11-17.

95 Hunt NC, James DS, Bull AD. The still video camera: a suitable and convenient method of demonstrating post mortem findings. Med Educ 1998;31:386-9.

96 Forrest ARW. Toxicological and biochemical analysis. In: Cotton DWK, Cross SS, eds. The hospital autopsy, 1st ed. Oxford: Butterworth Heinemann, 1993:134-43.

97 Hill RB, Anderson RE. The autopsy: medical practice and public policy, 1st ed. London: Butterworths, 1988.

98 Hutchins GM. Practice guidelines for autopsy pathology: autopsy reporting. Arch Pathol Lab Med 1995;119:123-30.

99 Leadbeatter S. Semantics of death certification. $7 \mathrm{R}$ Coll Physicians Lond 1986;292:129-32.

100 James DS, Bull AD. Information on death certificates: cause for concern? F Clin Pathol 1996;49:213-16. 